\title{
Cover illustration
More than just cool shades
}

magine trying to eat a poisonous basketball with spikes and sunglasses. That is the challenge a predator faces when confronted with the spiny puffer fish, Diodon holocanthus, illustrated on this month's cover.

The puffers are a group of nearly 100 species of mostly epipelagic fish, and are so named because of their ability to swell their bodies two to three times their original size until they are almost completely round.

The spiny puffer is perhaps the best known member of the families Tetraodontidae and Diodontidae, and has been studied in some detail. Perhaps only this species of all extant organisms combines the ability to inflate its body with the concomitant erection of nettlesome, pointed spines as a spectacular defence mechanism. The stomach can expand to nearly 100 times it original size by utilising extensive tissue pleating much like a kilt. Even the stomach pleats have pleats, and those pleats have pleats and so on. The puffer's body is prepared for this expansion by having a very flexible vertebral column that becomes concave towards the stomach when the stomach expands. Ribs are absent. The overlying skin is very elastic and, much like lycra, stretches as the stomach fills. With that stretching, the skin pulls taut and tension is placed on the struts of the previously flattened spines. These struts erect the spines and keep them rigid.

Enlarging the stomach dramatically increases internal pressure. Internal hydraulic pressure such as this should make it impossible to retain the water or air, and thus maintain the expanded ball-like shape. But these two closely related families of fish, which include triggerfish and sunfish, have another interesting modification to help maintain the higher internal pressure. Each member of these families has a small fluted mouth and an interesting oropharyngeal muscular complex. After the puffer fills its mouth with water, it uses this muscular system to force a plug-like valve from the base of the oropharynx against the front teeth creating a one way valve to prevent the water or air from being forced out of its mouth. The "plunger" muscular system then forces the water through the oesophagus and into the stomach and prevents extrusion.

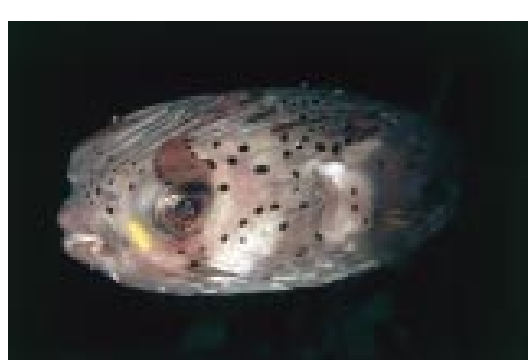

The "basketball with spines as armour" approach may not be enough defence, so this fish has another, more pedestrian, defence known in other prey species-poison. Tetrodotoxin is a vicious neurotoxin that is over 1000 times more potent than cyanide, can lead to respiratory arrest and circulatory failure in a few hours, and is concentrated in the skin, liver, intestines, and ovaries during certain times of the year. Curiously, this frightening heat stable toxin, which selectively blocks sodium channels as well as neuronal transmission, does not affect the puffer itself because of a rather slight genetic mutation in an amino acid sequence that prevents the blockade of the sodium channels by the toxin in these species. Believe it or not, humans actually eat this fish, and even call it a gourmet delicacy!

The puffer group holds another curious secret. The genome of this family is very small with genes and regulatory sequences of approximately 400 million base pairs compared to more than three billion in humans. This fish genome contains at least $90 \%$ commonality with the active human genome, although the human genome contains so much more extraneous genetic material. The study of this family of fish may help us understand why we mammals have so much extra DNA.

For us, though, this fish offers at least two peculiar twists in ocular development, and these can be seen clearly in the cover photo. This fish has orbital eyeshades that help block direct rays from the sun overhead. It also has corneal pigmentation presumably to reduce glare during diurnal foraging in its usual habitat of the shallow reef. Many other fish species have corneal pigment but this fish has another rarer form of apparent pigmentation, corneal iridescence. A few species of fish have been noted to have corneal iridescence although it has not been extensively studied in puffers. The iridescence in the corneas of puffers resembles that of several other fish with colouration created by stacks of multiple layers of connective tissue or collagen fibrils. These plates provide constructive interference and reflect a specific wavelength of light, and are particularly effective when the angle of incidence of light is oblique as bright downwelling sunlight would appear in a shallow reef. These reflected rays have a characteristic multicoloured appearance because only a restricted band of wavelengths is reflected for any single viewing angle. In the fish that have been studied, it has been calculated that the iridescent layer provides a significant increase in the visual range under water without sacrificing sensitivity (Lythgoe JN, Proc R Soc Lond B 1975;188:437-57).

The puffer, then, illustrates an iridescent layer in the cornea that is best seen from above, a tidy, compact genome, a poisonous neurotoxin, and the ability to become round like a football. That makes for more than just cool shades.

Ivan R Schwab

University of California Davis, Department of Ophthalmology, 4860 " $Y$ " Street, Suite 2400, Sacramento, CA 95817, USA; irschwab@ucdavis.edu 